\title{
Electrospun Nanofiber Membranes Containing Molecularly Imprinted Polymer (MIP) for Rhodamine B (RhB)
}

\author{
Haiqing Liu, Xiulan Lei, Yunyun Zhai, Lei Li* \\ College of Biological, Chemical Science and Engineering, Jiaxing University, Jiaxing, China \\ Email: "lei.li@mail.zjxu.edu.cn
}

Received February 23, 2012; revised March 21, 2012; accepted April 12, 2012

\begin{abstract}
A simple method for the formation of molecularly imprinted membrane of Rhodamine $\mathrm{B}(\mathrm{RhB})$ was developed by electrospinning. RhB molecularly imprinted microspheres were produced by precipitation polymerization using $\mathrm{RhB}$, acrylamide, ethylene glycol dimethacrylatea (EGDMA), azobisisobutyronitrile (AIBN) and acetonitrile as template, functional monomer, cross-linking agent, initiator and porogen, respectively. Then molecularly imprinted membranes (MIMs) were produced via electrospinning technique with polyethylene terephthalate (PET) as the matrix polymer. The as-prepared nanofiber membranes were characterized by scanning electron microscopy (SEM). Optimization studies with the aim to enhance the MIP selection adsorption were carried out with respect to the amount of membrane, $\mathrm{pH}$ and adsorption time. Linear range and detection limit were $0.01-20 \mu \mathrm{mol} / \mathrm{L}$ and $2.0 \times 10^{-3} \mu \mathrm{mol} / \mathrm{L}$, respectively. HPLC analysis showed that in the optimized conditions of separation and enrichment, the recovery rate can reach $97.8 \%$ $117.1 \%$, relative standard deviation $(\mathrm{n}=3)$ was $1.36 \%-2.19 \%$ in employing MIMs to the RhB simulated water samples. The results showed that the imprinted polymer exhibited higher affinity for Rhodamine B compared to non-molecularly imprinted polymers membranes (NIMs) and molecularly imprinted particles (MIP).
\end{abstract}

Keywords: Molecularly Imprinted Membrane; Electrospinning; Precipitation Polymerization; Rhodamine B; Separation and Enrichment

\section{Introduction}

Molecularly imprinted polymers are obtained by functional monomers and cross-linking monomers around a template molecule, leading to a highly three-dimensional cross-linked network polymer [1,2]. When polymerization by covalent, non-covalent or semi-covalent approaches has taken place and template molecule is removed. MIPs with binding sites with shape, size and functionalities complementary to the target analytes are established. MIPs have an unusual recognition of their corresponding target molecules $[3,4]$. Because of the selectivity, easy preparation and economic, MIPs are ideal materials and the recent concerns! The MIPs are stable, robust and resistant to a wide range of $\mathrm{pH}$, solvents, thermal stability, and have good specific adsorption properties and selectivity ability. It has been widely used for selective enrichment and separation in environmental applications [5-7], food applications [8], chemical sensors [9-11], optical sensors [12], protein selections $[13,14]$, and pharmaceutical analysis [15,16]. However there are several limitations and challenges with the rapid

\footnotetext{
"Corresponding author.
}

development of MIPs. For example, 1) MIPs are usually very small particles and hard to accumulate during the experiment; 2) the process column needs to be filled and large consumption of solvents; 3 ) some active sites were also embedded into the particles in the traditional methods. So the methods and strategies of easy preparing MIPs with much larger surface areas are important and urgent.

The ability to create materials with well-controlled nanofibers is of intense interest for a variety of applications. In recent years, electrospinning technology has gained widespread attention since it is known to be an effective fabrication tool for preparing various polymeric nanofibers and nanostructured materials with a high aspect ratio and high surface area [17-20] (Scheme 1). So electrospun nanofibers are good candidates for the adsorption and filtration because of their high permeability, large surface area and small pore size. In addition, the electrospun nanofibers are easy to assemble, modify and process into different applications, such as biomedicine [21], tissue engineering [22] and armored fabric.

Furthermore, the introduction of MIP on the surface of 


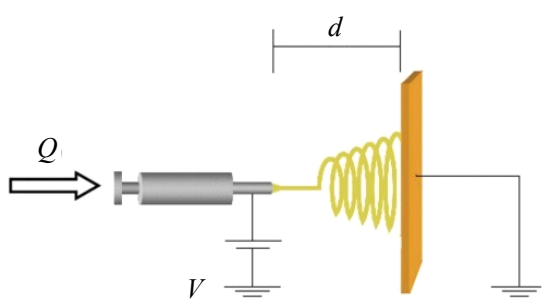

Scheme 1. Schematic diagram of a laboratory basic setup for an electrospinning experiment with a horizontal arrangement of the electrodes.

an electrospun nanofiber membrane enables its utilization as an adsorbent in separation and accumulation for complex sample treatment. Keiichi Yoshimatsu and Lei Ye found that molecularly imprinted nanoparticles were encapsulated into polymer nanofibers with electrospinning method [23]. While Ioannis S. Chronakis and Lei Ye prepared encapsulated and selective recognition of molecularly imprinted theophylline and estradiol nanoparticles within electrospun polymer nanofibers [24]. Silvia Piperno described the polymer nanofibers with entrapped molecularly imprinted polymer (MIP) nanoparticles and studied their possible use in a fluorescence-based biosensor application [25]. Compared to encapsulation for nanoparticles, there are some advantages for molecularly imprinted polymers to dissolve into uniform and homogeneous electrospinning solutions. So Ioannis S. Chronakis and Lei Ye prepared smooth electrospun poly-(ethylene terephthalate) polymer which contained molecular recognition sites for 2,4-dichlorophenoxyacetic acid [26]. The electrospun nanofibers provided a porous matrix with very high surface area-to-volume ratio, which is desirable for the selective rebinding of the target molecule. They demonstrated for the first time that electrospun template directed molecular imprinting is a viable method for creating molecularly imprinted nanofibers.

Rhodamine $\mathrm{B}(\mathrm{RhB})$ is a kind of peach red synthetic dye. It is widely used in industry, such as textile, plastics, food, dyeing, paper making and printing. As a consequence of its solubility, high chemical and biological stability, RhB has been affected to environment $[27,28]$. It is well known, RhB also is harmful to skin, eyes and brain. So nowadays there are many ways to remove $\mathrm{RhB}$, such as condensation, sink to the bottom, adsorption [29, 30], reversed-phase permeability, ion exchange and chemical oxidation [31]. But there is no mature and effective method to separate and accumulate RhB in some complex samples, such as food, environmental water samples. So we choose RhB as our research objective to develop some economical and effective separation ways!

Therefore, we use the precipitation polymerization to make $\mathrm{RhB}$ molecularly imprinted polymer particles. Then these MIMs through electrospinning method were made. The MIMs were used for separation and accumulation of $\mathrm{RhB}$. The results showed that the imprinted polymer exhibited higher affinity compared to molecularly imprinted particles. Meanwhile it is necessary to identify several optimal conditions with respect to maximum separation performances.

\section{Experiment Section}

\subsection{Materials and Instrumentations}

Acrylamide, ethylene glycol dimethacrylatea (EGDMA), azobisisobutyronitrile (AIBN), acetonitrile and polyethylene terephthalate (PET) were purchased from J\&K Scientific Ltd. Rhodamine B (RhB) was obtained from Sinopharm Chemical Reagent Co. All solvents were obtained from Sinopharm Chemical Reagent Co. and used as received. All aqueous solutions were prepared using purified water with a resistance of $18.2 \mathrm{M} \Omega \cdot \mathrm{cm}$.

Field emission scanning electron microscopy (FESEM) was obtained by Hitachi S-4800 (S-4800, Hitachi Ltd., Japan). The HPLC (Diane 3000) and UV/Vis (Shimadzu 2550) were used for analyses. High voltage direct current generator (JG50-1) was bought from Shanghai Shenfa Ltd.

\subsection{Synthesis of the Electrospinning MIP Membranes}

\subsubsection{Preparation of Molecularly Imprinted Polymers (MIPs) Microspheres}

A mixture containing $0.5 \mathrm{mmol} \mathrm{RhB}, 3 \mathrm{mmol}$ Acrylamide, 15 mmol EGDMA and $100 \mathrm{mg}$ AIBN were placed in a glass vial. After ultrasonic mixing for $5 \mathrm{~min}$, the mixtures were purged with nitrogen for $15 \mathrm{~min}$. The polymerization proceeded at $60^{\circ} \mathrm{C}$ for $24 \mathrm{~h}$. Then the template molecules were washed away by mixed solvent (acetic acid: methanol $=1: 9$ (volume)), until no $\mathrm{RhB}$ could be detected in the washing solutions using UV/Vis spectroscopy. After vacuum drying, MIP microspheres were obtained.

\subsubsection{Preparation of Molecularly Imprinted Membranes (MIMs) via Electrospinning}

Polyethylene terephthalate (PET) solution was prepared by adding PET $(1.0 \mathrm{~g})$ into trifluoroacetic acid (TFA) $(4.0 \mathrm{~mL})$ with continuous agitation in close vials for $3 \mathrm{~h}$ at room temperature. Then the solution of molecularly imprinted polymers in the dichloromethane was blended into PET solution. After agitation and ultrasonication for $2 \mathrm{~h}$, the resultant homogeneous solutions were fed into a plastic syringe driven by a syringe pump (KDS-200, Focus Co., Ltd., USA). The positive electrode of a high voltage power supply was clamped to the metal needle $(0.8 \mathrm{~mm})$ tip of the syringe. The cylindrical collector 
covered by aluminum foil was used for collector. The applied voltage was $18 \mathrm{KV}$, and the tip-to-collector distance was $15 \mathrm{~cm}$. The ambient temperature and relative humidity were maintained at $25^{\circ} \mathrm{C}$ and $45 \%$, respectively. The prepared fibrous membranes were dried in vacuum at room temperature to remove the trace solvent. NIMs were prepared by the same process without $\mathrm{RhB}$ for template. The morphology and size of polymer microspheres and electrospinning membranes were obtained by scanning electron microscopy.

\subsection{Analytical Conditions}

\subsubsection{Optimization of the Amount of MIP Membranes}

Molecularly imprinted polymer membranes of $10.0 \mathrm{mg}$, $20.0 \mathrm{mg}, 30.0 \mathrm{mg}, 40.0 \mathrm{mg}, 50.0 \mathrm{mg}$ and $60.0 \mathrm{mg}$ were added into $\mathrm{RhB}$ aqueous solution $(40 \mu \mathrm{mol} / \mathrm{L}, 10 \mathrm{~mL})$. Then the sample was detected and analyzed after slight shock in continuous rotary oscillator for $2 \mathrm{~h}$. The solution of $10 \mu \mathrm{L}$ was removed for the analysis with HPLC each time.

\subsubsection{Effects of $\mathbf{p H}$}

BR (Britton-Robinson) buffer solution was prepared with different $\mathrm{pH}$ values to research $\mathrm{RhB}$ adsorption situation.

\subsubsection{Optimization of Adsorption Time}

To investigate the adsorption time, molecular imprinted polymer membranes were added into Rhodamine B aqueous solutions on the rotary oscillator to slightly shock. From the beginning time, a sample was taken to detect and analysed with HPLC every $10 \mathrm{~min}$. NIMs were also investigated in the same method to determine the adsorption time compared to the corresponding MIMs.

\subsubsection{Static Adsorption}

After optimization of the amount of membrane, $10.0 \mathrm{mg}$ MIMs were selected as adsorbents in the process of the static adsorption experiments. MIMs were added into Rhodamine $\mathrm{B}$ aqueous solution with different concentrations $(1-40 \mu \mathrm{mol} / \mathrm{L})$. After slightly shocked at room temperature in the rotary oscillator for $2 \mathrm{~h}$, the samples were analysed by HPLC. NIMs were also studied in the same method.

\subsubsection{Chromatographic Conditions}

The concentrations of Rhodamine B solutions were assayed on UltiMate 3000 high performance liquid chromatography (HPLC; Diane, USA). The HPLC system equipped with a G1314B UV/Vis detector and G1328B manual-sampler. The stationary phase consisted of a column $(5 \mu \mathrm{m}, 4.6 \mathrm{~mm} \times 250 \mathrm{~mm})$ packed with ex- tend- $\mathrm{C}_{18}$. The detection wavelength was $550 \mathrm{~nm}$ for Rhodamine B. The mobile phase consisted of watermethanol $(25: 75, \mathrm{v} / \mathrm{v})$ for adsorption, water-methanol $(28: 72, \mathrm{v} / \mathrm{v})$ for $\mathrm{pH}$ effect, and water-methanol $(65: 35$, $\mathrm{v} / \mathrm{v}$ ) for river water sample. The column was eluted at a flow rate of $1.0 \mathrm{~mL} / \mathrm{min}$ at $35^{\circ} \mathrm{C}$.

\subsubsection{Recovery Experiments}

Samples with high, medium and low concentrations were prepared by the stock solution $(6 \mu \mathrm{mol} / \mathrm{L}$ of $\mathrm{RhB})$ and RhB simulated water samples. $70 \%, 50 \%, 30 \%$ of the amount of reference substance stock solutions $(6 \mu \mathrm{mol} / \mathrm{L})$ were added into water samples $(20 \mathrm{~mL})$ to make recovery experiments separately for the imprinted membrane and blank membrane.

MIMs $(10.0 \mathrm{mg})$ were collected to place into conical flask. Then add to the high, medium and low concentration samples. After slight shock for $2 \mathrm{~h}$ at room temperature in a rotary shock device, liquid chromatography was used to determine the equilibrium adsorption concentration of the solution. According to the concentration of the solution before and after adsorption, the changes in the molecularly imprinted polymer membranes for the adsorption of $\mathrm{RhB}$ target molecule were calculate. The same method was used to determine the adsorbed NIMs.

Unknown concentrations of RhB simulated water sample was made in the range of standard curve. Through pre-treatment with the imprinted membrane, the concentration of samples was detected by liquid chromatography. At the same time NIMs were used to determine and then compared with the imprinted membrane.

\section{Results and Discussion}

\subsection{Preparation of Molecularly Imprinted Microspheres}

Precipitation polymerization was used to prepare molecularly imprinted microspheres (Scheme 2). In the reaction system, surface of prepared polymer microspheres was clean because of without the stabilizing agents or surfactants. SEM observation found that the polymer microspheres were spherical, and were arranged in an orderly three-dimensional shape. There is only a little bonding between the particles (Figure 1). The diameters of molecularly imprinted microspheres (a) and nonmolecularly imprinted microspheres (b) were about 1 $\mu \mathrm{m}$.

\subsection{Preparation of Molecularly Imprinted Membranes via Electrospinning}

In our work, PET solution prepared by adding PET into trifluoroacetic acid (TFA) with continuous agitation was used for electrospinning. PET was selected as the sup- 

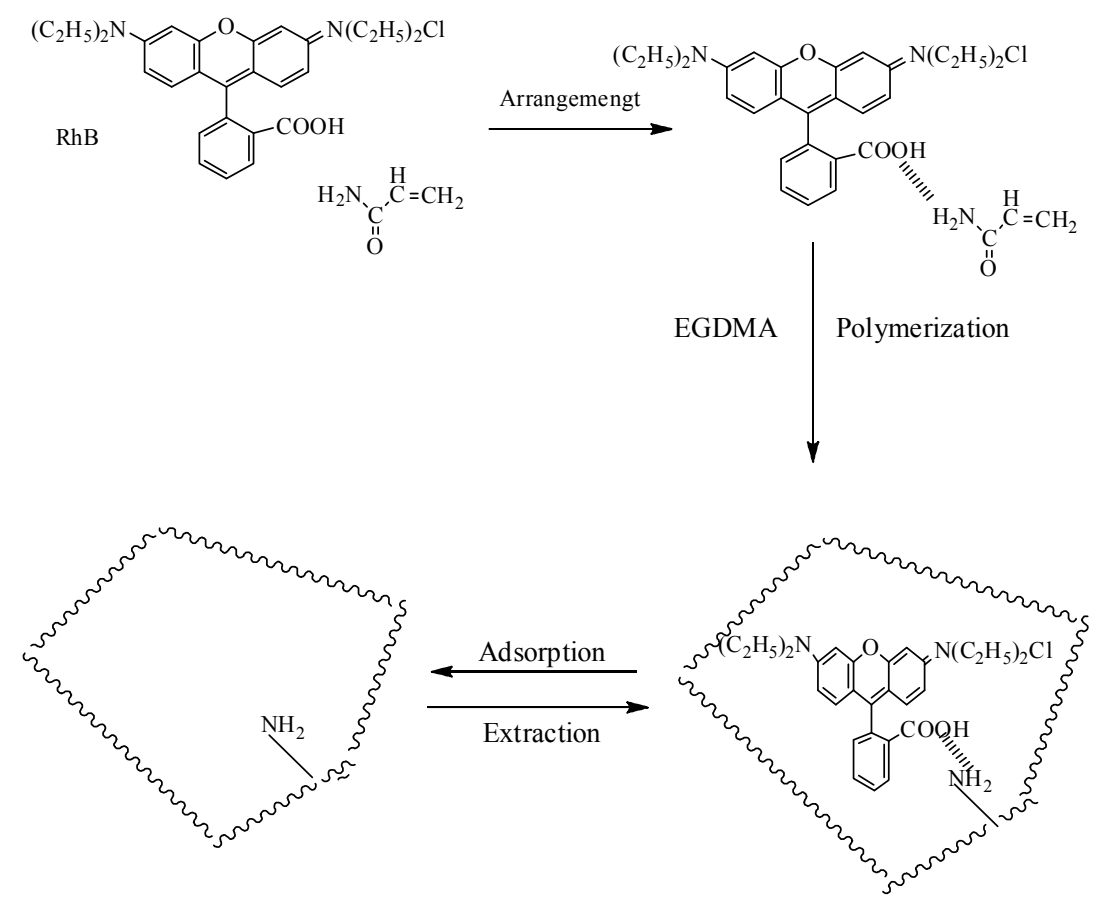

Scheme 2. Schematic diagram of the molecular imprinting process for RhB.

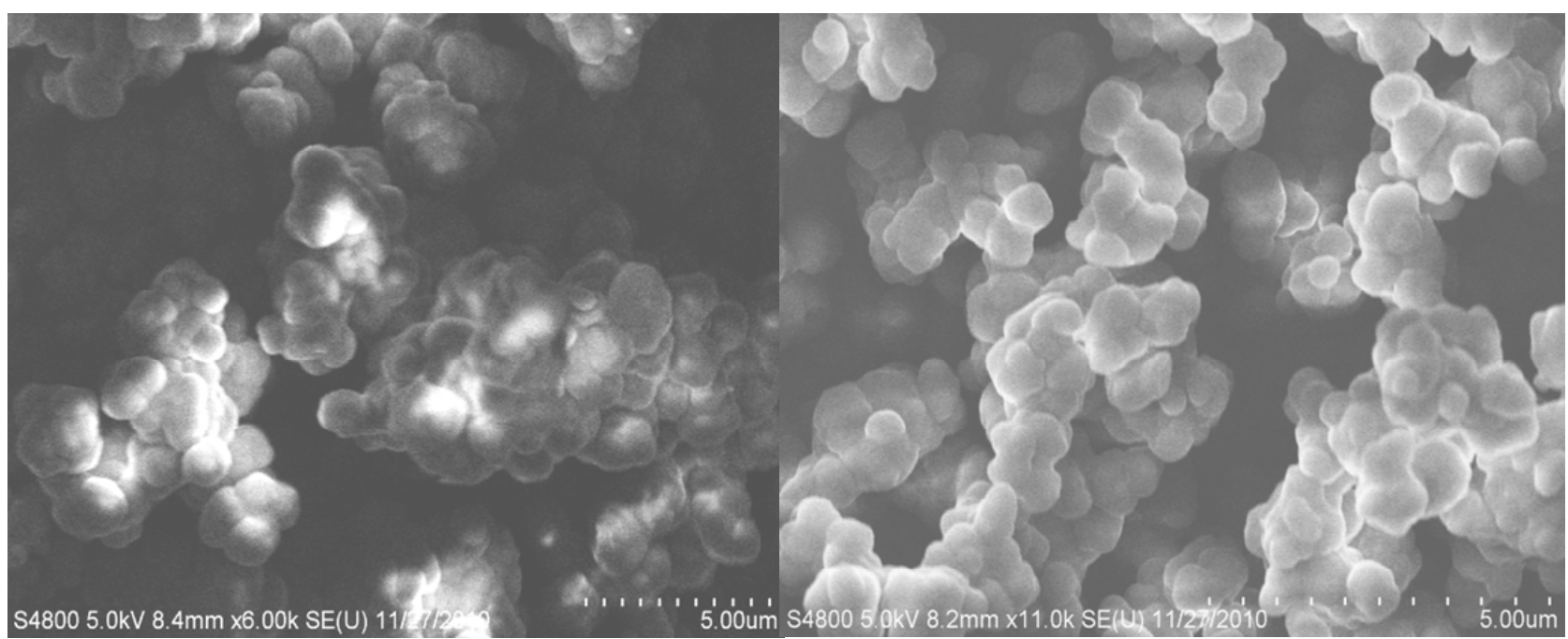

(a)

(b)

Figure 1. SEM images of molecularly imprinted microspheres (a) and non-molecularly imprinted microspheres (b).

porting fiber matrix because it could be easily electrospun to ultrafine nanofibers [26]. The solution of molecularly imprinted polymer (MIP) in the dichloromethane was blended into PET solution. SEM observation found that the MIP wire and blank NIP wire show smooth surfaces and good morphology. The long, electrospun nanofibers were randomly distributed with very uniform and dense structures. The fibers exhibited good morphology with an average fiber diameter in the range of 100 - $200 \mathrm{~nm}$ (Figure 2). The addition of RhB template molecule to the starting polymer solution caused no noticeable change in morphology in the nanofibers. It was based on the fact that the electrospinning started from a homogeneous polymer solution containing PET, and $\mathrm{RhB}$ template molecularly imprinted microspheres.

\subsection{Adsorption Experiment}

In order to future applications, we were interested in studying $\mathrm{RhB}$ binding in an aqueous environment at a low concentration level. Adsorption experiments were operated by MIM or NIM at different concentrations of 


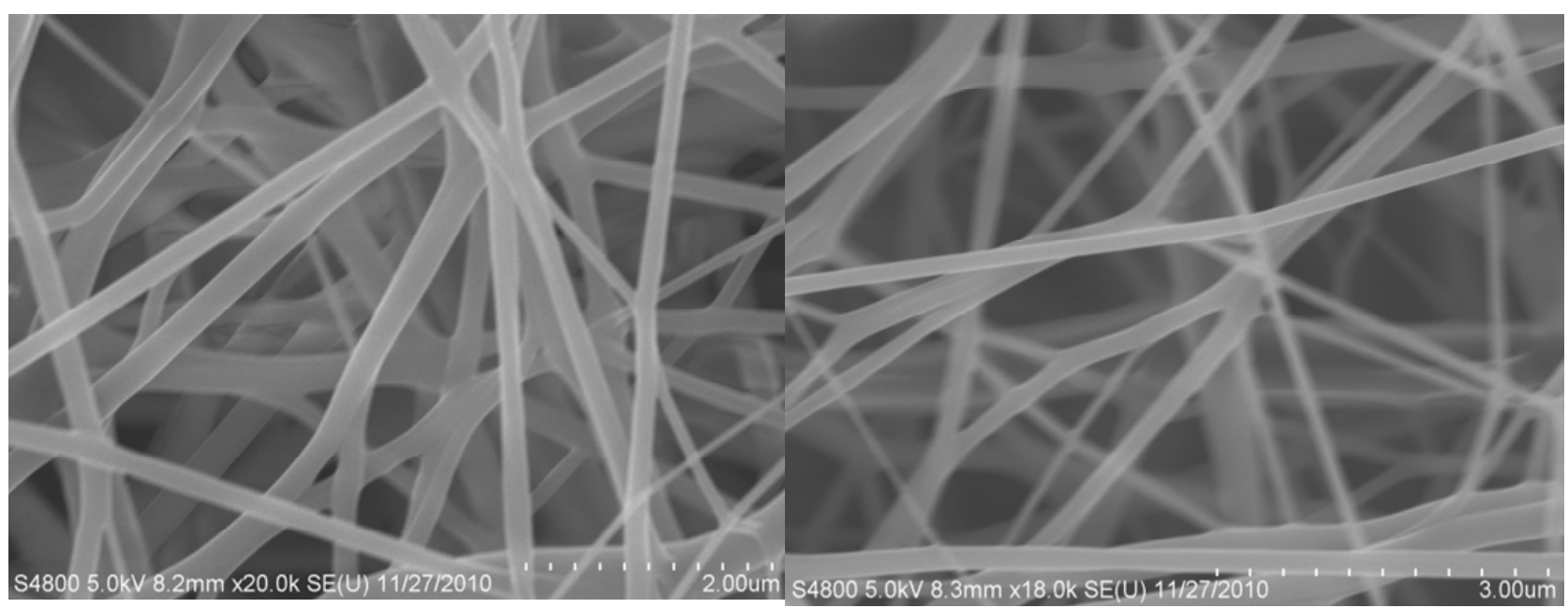

(a)

(b)

Figure 2. SEM images of electrospinning MIMs (a) and NIMs (b).

target molecules (or template molecules), respectively.

The adsorption capacity $Q$ is calculated by the following formula:

$$
Q=\frac{\left(C_{0}-C\right) \times V}{m}
$$

where $Q$ is the static equilibrium adsorption capacity $(\mathrm{mg} / \mathrm{g}) ; C_{0}$ is the initial substrate concentration $(\mathrm{mg} / \mathrm{L}) ; C$ is the substrate concentration $(\mathrm{mg} / \mathrm{L})$ at equilibrium; $V$ is the volume of substrate solution (L); $m$ is the amount of added polymer film (g).

\subsubsection{Optimization of the Amount of MIMs}

Optimization of the amount of film is to examine the optimum amount of film to achieve the province of the material and manpower. From Figure 3, we can see in the same conditions, the adsorption capacities were reduced with the increase of the membrane quality. The amount of $10 \mathrm{mg}$ MIMs was selected in the following experiments based on the economic point.

\subsubsection{Effects of $\mathbf{p H}$}

RhB solutions with different $\mathrm{pH}(3$ - 10) were prepared to research $\mathrm{RhB}$ adsorption situation. Then $10 \mathrm{mg}$ MIMs was added into the solution. The samples were collected after slightly shake for $2 \mathrm{~h}$. Then UltiMate 3000 series of HPLC (UV detector) was used for detection of RhB. In Figure 4, the results showed that the adsorption of RhB is affected by $\mathrm{pH}$.

Carboxyl was damage by esterification under acidic conditions $(\mathrm{pH}<7)$. Conversely the hydrogen bond of the amino was lost under alkaline conditions $(\mathrm{pH}>7)$. So the recognition sites on the polymer were destroyed. And then the adsorption properties of the MIMs were affected. So the neutral condition $(\mathrm{pH}=7)$ is the best conditions in

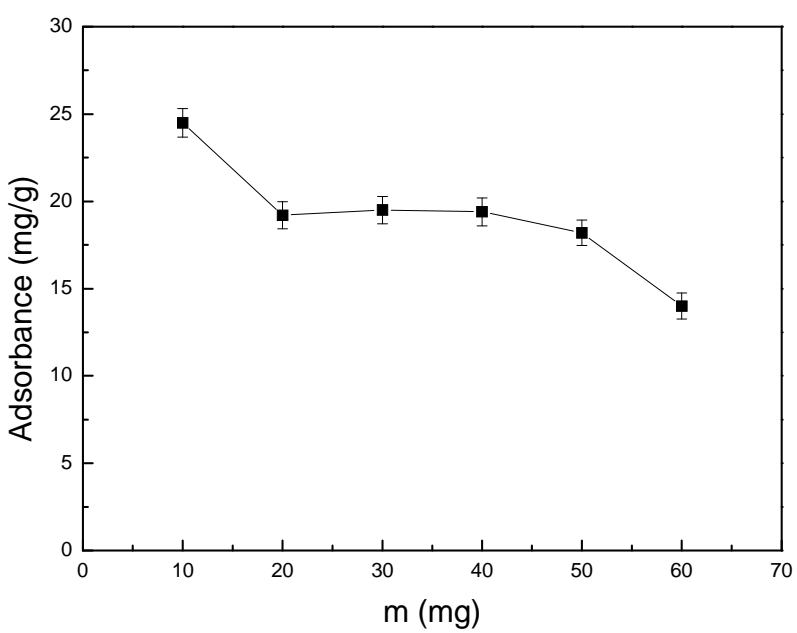

Figure 3. The dosage optimization of MIMs for adsorbance of RhB.

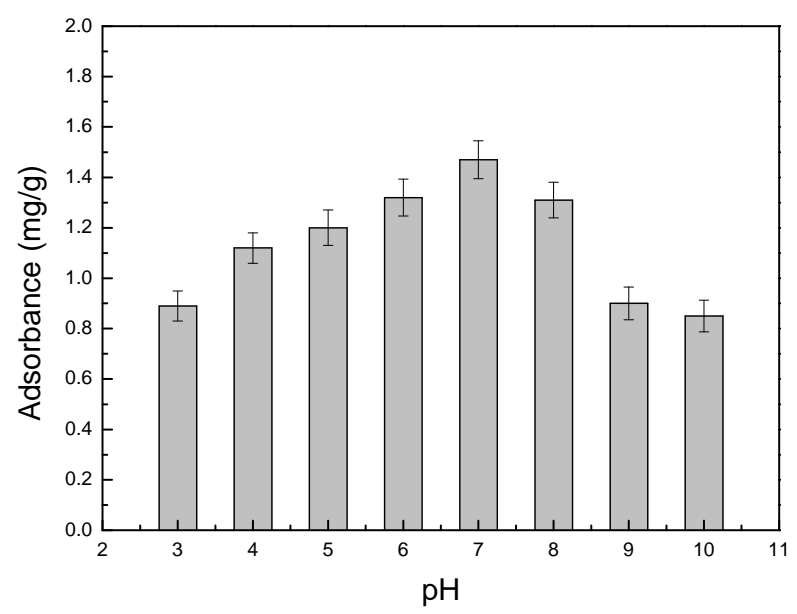

Figure 4. The dependence between adsorbance and $\mathrm{pH}$ for MIMs in absence of RhB. 
the later studies.

\subsubsection{Optimization of Adsorption Time}

Optimization of adsorption time was to determine the best time in adsorption process. From Figure 5, the adsorption of MIMs was superior to the NIMs and had higher recognition ability than NIMs. At the same time, MIMs and NIMs has a desorption phenomenon after the slightly shock for $80 \mathrm{~min}$. So the samples were extracted after slightly shock for $80 \mathrm{~min}$ for analysis in the followup work.

\subsubsection{Static Adsorption}

From Figure 6, we can see with the concentration increase of Rhodamine $\mathrm{B}$, the adsorption of $\mathrm{RhB}$ was increased by the same quality of Rhodamine B MIMs. Meanwhile the adsorption of RhB of MIM was obviously superior to the NIMs. So this phenomenon can illustrate that the MIMs contains a lot of specific binding sites.

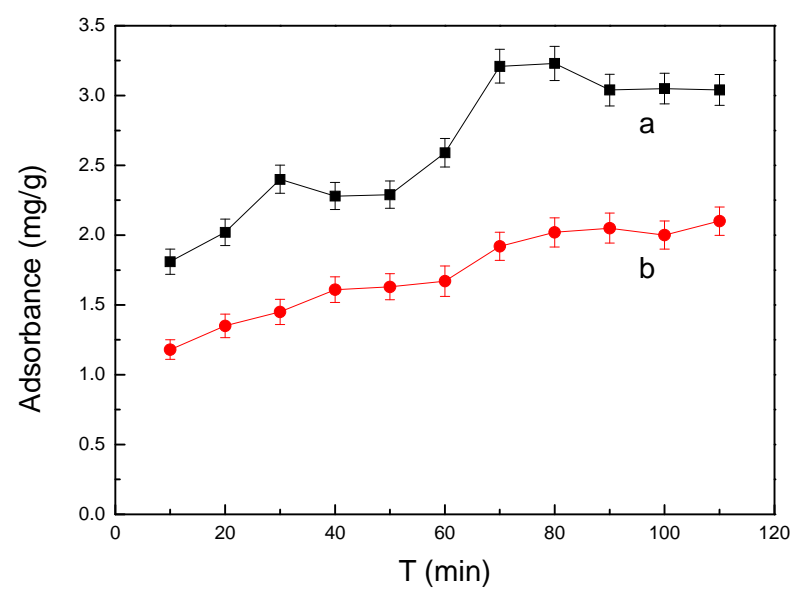

Figure 5. The dependence between adsorbance and time for MIMs (a) and NIMs (b) in absence of RhB.

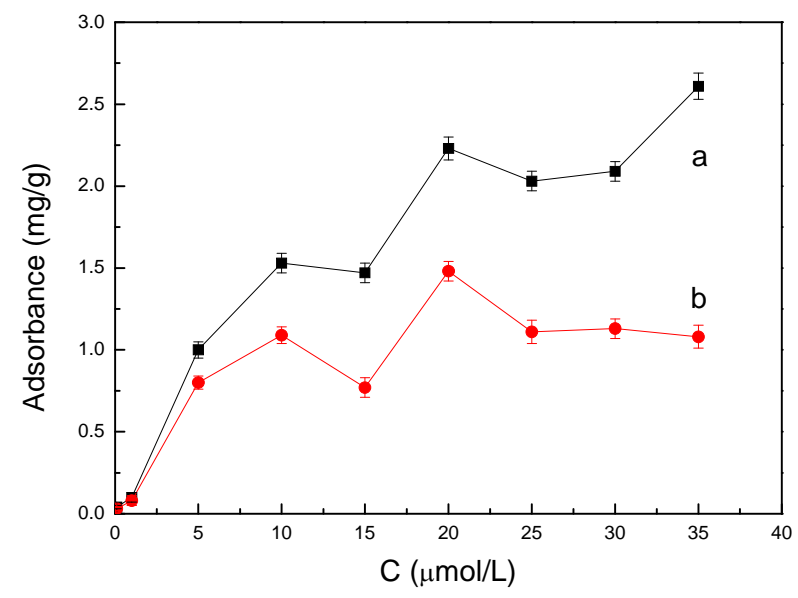

Figure 6. The dependence between adsorbance and concentrations of RhB for MIMs (a) and NIMs (b).

\subsubsection{Regeneration of MIMs}

The stability and reproducibility of MIMs were investigated. As shown in Figure 7, the adsorption of MIMs for $\mathrm{RhB}$ was not significantly reduced after eluting several times $(n>5)$ with pure methanol. Therefore, the results show that MIMs has good regenerative adsorption and availability.

\subsubsection{Elution and Elution Volume}

Different eluents have different eluotropic capacities for target molecules. This paper studied the eluotropic capacities of methanol, acetonitrile and water. The eluotropic capacities between methanol and acetonitrile were equivalent. Because of the toxicity of acetonitrile, methanol is better than acetonitrile. From the other side of eluotropic time, the eluotropic time of methanol $(0.5 \mathrm{~h})$ is shorter than water $(2 \mathrm{~h})$. Therefore, from the above considerations, the chromatographic pure methanol acted as eluent in the experiment.

\subsection{Analytical Performance}

\subsubsection{Standard Curve and Detection Limit}

A series of low concentration of the $\mathrm{RhB}$ standard solution were prepared by using solution of $\mathrm{RhB}$, which analyzed by HPLC method with the optimized chromatographic conditions. And the standard curve could be expressed by the peak area (A) on the concentration $(\mu \mathrm{g} / \mathrm{mL})$. The results indicated that the linearity was investigated at the range of $0.01-20 \mu \mathrm{mol} \cdot \mathrm{L}^{-1}$ with squared coefficients of correlation $\mathrm{R}=0.9996$. And the linear equation was $\mathrm{A}=1.91 * \mathrm{C}-0.2$. Additionally, the detection limit (LOD; $3 \sigma / K, n=11$ ) for $\mathrm{RhB}$ was found to be $2 \mathrm{nmol} \cdot \mathrm{L}^{-1}$.

\subsubsection{Evaluation of Method}

The high, medium and low concentrations of three dif-

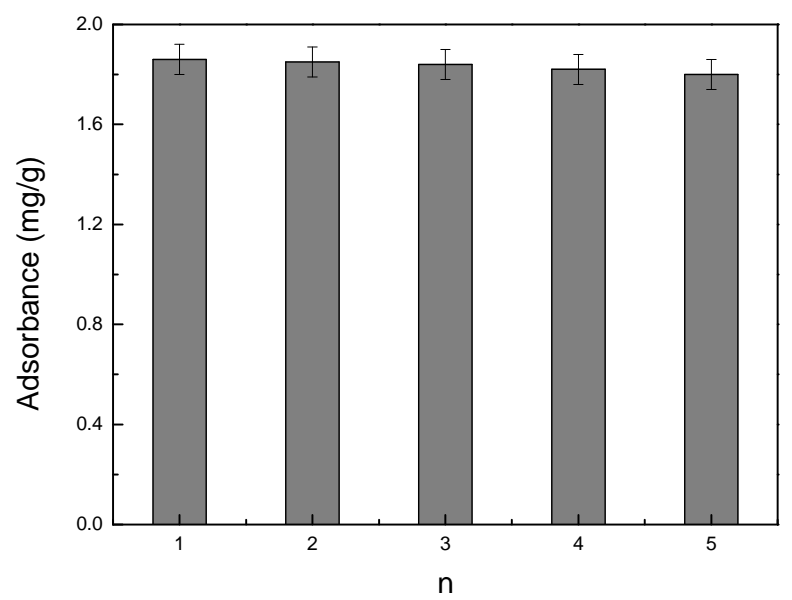

Figure 7. The adsorbance changes between different applications for the same MIMs for RhB. 
ferent standard RhB solutions were added in the river water samples. MIMs or NIMs was used to enrichment the $\mathrm{RhB}$ in river water samples for $1 \mathrm{~h}$ with the optimal experimental conditions. Then the MIM or NIM was washed with methanol for three times. And the spiked recoveries were determined by HPLC. From Table 1, the recovery of MIMs for RhB was $97.80 \%-117.2 \%$ and the relative standard deviation $(\mathrm{N}=3)$ was $1.4 \%-8.7 \%$. HPLC results showed that there is no $\mathrm{RhB}$ in river water sample. And the recovery of MIMs is obviously higher than NIMs. So MIM has good selective adsorption for RhB than NIMs.

\section{Conclusion}

In this paper molecularly imprinted polymer particles of Rhodamine B was synthesized. Then molecularly imprinted membranes (MIMs) were made by electrospinning technology. MIMs were applied to enrichment and separation of RhB. All results show that MIMs have good selective adsorption than NIMs and achieve the desired effect. The above results demonstrate that electrospun molecular imprinting is a viable method for creating robust molecularly imprinted nanofibers that can selectively the RhB target molecule. The imprinted nanofibers also had a well-defined morphology and physical stability. Considering nanofiber processing, the present electrospinning-imprinting method is promising for its simple operation and high efficiency. Meanwhile, we have prepared the magnetic molecularly imprinted polymers using atrazine as a template on surface modification of the $\mathrm{Fe}_{3} \mathrm{O}_{4} @ \mathrm{SiO}_{2}$ nanoparticles [32]. So we are designing the molecularly imprinted membrane based on the electrospinning technology and magnetic materials.

\section{Acknowledgements}

This work was financially supported by the Natural Sci-

Table 1. The recovery of standard addition and relative standard deviation (RSD) with RhB in river water sample.

\begin{tabular}{cccccc}
\hline $\begin{array}{c}\text { Solid phase } \\
\text { extraction } \\
\text { materials }\end{array}$ & $\begin{array}{c}\text { Blank } \\
\text { sample } \\
\text { solution }\end{array}$ & $\begin{array}{c}\text { Addition } \\
(\mu \mathrm{mol} / \mathrm{L})\end{array}$ & $\begin{array}{c}\text { Detection } \\
(\mu \mathrm{mol} / \mathrm{L})\end{array}$ & $\begin{array}{c}\text { Recovery } \\
(\%)\end{array}$ & $\begin{array}{c}\mathrm{RSD} \\
(\%)\end{array}$ \\
\hline MIMs & $\mathrm{ND}^{*}$ & 1.2 & 1.17 & 97.80 & 2.2 \\
& & 0.6 & 0.70 & 117.2 & 1.6 \\
& & 2.0 & 2.11 & 105.4 & 1.4 \\
NIMs & $\mathrm{ND}^{*}$ & 1.2 & 0.67 & 72.65 & 4.1 \\
& & 2.0 & 1.27 & 63.63 & 8.7 \\
\hline
\end{tabular}

$\mathrm{ND}^{*}:$ No detected. ence Foundation of China (No. 21177049, No. 51103063), the Zhejiang Provincial Natural Science Foundation (No. Y4110545), the Program for Science and Technology of Zhejiang (No. 2011C22096, No. 2011C37033), the Zhejiang Science and Technology Innovation Project for university student (2011R417020) and the Program for Science and Technology of Jiaxing (No. 2010AY1081, No. 2011AY1028, No. 2011AY1007).

\section{REFERENCES}

[1] L. Ye and K. Mosbach, "Molecular Imprinting: Synthetic Materials as Substitutes for Biological Antibodies and Receptors," Chemical Materials, Vol. 20, No. 3, 2008, pp. 859-868. doi:10.1021/cm703190w

[2] C. Alexander, H. S. Andersson, L. I. Andersson, R. J. Ansell, N. Kirsch, I. A. Nicholls, J. O’Mahony and M. J. Whitcombe, "Molecular Imprinting Science and Technology: A Survey of the Literature for the Years Up to and Including 2003," Journal of Molecular Recognition, Vol. 19, No. 2, 2006, pp. 106-180. doi:10.1002/jmr.760

[3] J. Fan, Y. Wei, J. Wang, C. Wu and H. Shi, "Study of Molecularly Imprinted Solid-Phase Extraction of Diphenylguanidine and Its Structural Analogs," Analytica Chimica Acta, Vol. 639, No. 1-2, 2009, pp. 42-50. doi:10.1016/j.aca.2009.02.045

[4] H. Yan, K. H. Row and G. Yang, "Water-Compatible Molecularly Imprinted Polymers for Selective Extraction of Ciprofloxacin from Human Urine," Talanta, Vol. 75, No. 1, 2008, pp. 227-232.

doi:10.1016/i.talanta.2007.11.002

[5] Y. Li, X. Li, J. Chu, C. Dong, J. Qi and Y. Yuan, "Synthesis of Core-Shell Magnetic Molecular Imprinted Polymer by the Surface RAFT Polymerization for the Fast and Selective Removal of Endocrine Disrupting Chemicals from Aqueous Solutions," Environmental Pollution, Vol. 158, No. 7, 2010, pp. 2317-2323. doi:10.1016/j.envpol.2010.02.007

[6] Y. Ji, J. Yin, Z. Xu, C. Zhao, H. Huang, H. Zhang and C. Wang, "Preparation of Magnetic Molecularly Imprinted Polymer for Rapid Determination of Bisphenol A in Environmental Water and Milk Samples," Analytical and Bioanalytical Chemistry, Vol. 395, No. 4, 2009, pp. 1125 1133. doi.10.1007/s00216-009-3020-5

[7] W. Guo, W. Hua, J. Pan, H. Zhou, W. Guan, X. Wang, J. Dai and L. Xu, "Selective Adsorption and Separation of BPA from Aqueous Solution Using Novel Molecularly Imprinted Polymers Based on Kaolinite $/ \mathrm{Fe}_{3} \mathrm{O}_{4}$ Composites," Chemical Engineering Journal, Vol. 171, No. 2, 2011, pp. 603-611. doi:10.1016/j.cej.2011.04.036

[8] H. Zhu, L. Ma, G. Fang, M. Pan, J. Lu, X. Wang and S. Wang, "Preparation of a Molecularly Imprinted Polymer Using TMB as a Dummy Template and Its Application as SPE Sorbent for Determination of Six PBBs in Water and Fish Samples," Anal Methods, Vol. 3, No. 2, 2011, pp. 393-399. doi: 10.1039/c0ay00479k

[9] D. Lakshmi, A. Bossi, M. J. Whitcombe, I. Chianella, S. 
A. Fowler, S. Subrahmanyam, E. V. Piletska and S. A. Piletsky, "Electrochemical Sensor for Catechol and Dopamine Based on a Catalytic Molecularly Imprinted Polymer-Conducting Polymer Hybrid Recognition Element," Analytical Chemistry, Vol. 81, No. 9, 2009, pp. 3576-3584. doi: $10.1021 / \mathrm{ac} 802536 \mathrm{p}$

[10] R. N. Liang, D. A. Song, R. M. Zhang and W. Qin, "Potentiometric Sensing of Neutral Species Based on a Uniform-Sized Molecularly Imprinted Polymer as a Receptor," Angewandte Chemie International Edition, Vol. 49, No. 14,2010 , pp. 2556-2559. doi: 10.1002/anie. 200906720

[11] X. Hu, Q. An, G. Li, S. Tao and J. Liu, "Imprinted Photonic Polymers for Chiral Recognition," Angewandte Chemie International Edition, Vol. 45, No. 48, 2006, pp. 8145-8148. doi:10.1002/anie.200601849

[12] A. L. Medina-Castillo, G. Mistlberger, J. F. FernandezSanchez, A. Segura-Carretero, I. Klimant and A. Fernandez-Gutierrez, "Novel Strategy to Design Magnetic, Molecular Imprinted Polymers with Well-Controlled Structure for the Application in Optical Sensors," Macromolecules, Vol. 43, No. 1, 2010, pp. 55-61. doi:10.1021/ma902095s

[13] L. Li, X. He, L. Chen and Y. Zhang, "Preparation of Core-shell Magnetic Molecularly Imprinted Polymer Nanoparticles for Recognition of Bovine Hemoglobin," Chemistry-An Asian Journal, Vol. 4, No. 2, 2009, pp. 286-293. doi:10.1002/asia.200800300

[14] T. Jing, H. Du, Q. Dai, H. Xia, J. Niu, Q. Hao, S. Mei and Y. Zhou, "Magnetic Molecularly Imprinted Nanoparticles for Recognition of Lysozyme," Biosensors and Bioelectronics, Vol. 26, No. 2, 2011, pp. 301-306. doi:10.1016/j.bios.2010.08.044

[15] L. Chen, S. Xu and J. Li, "Recent Advances in Molecular Imprinting Technology: Current Status, Challenges and Highlighted Applications," Chemical Society Reviews, Vol. 40, No. 5, 2011, pp. 2922-2942. doi: $10.1039 / \mathrm{c} 0 \mathrm{cs} 00084 \mathrm{a}$

[16] L. Chen, X. Zhang, L. Sun, Y. Xu, Q. Zeng, H. Wang, H. $\mathrm{Xu}, \mathrm{A}$. Yu, H. Zhang and L. Ding, "Fast and Selective Extraction of Sulfonamides from Honey Based on Magnetic Molecularly Imprinted Polymer," Journal of Agricultural and Food Chemistry, Vol. 57, No. 21, 2009, pp. 10073-10080. doi:10.1021/jf902257d

[17] D. Li and Y. Xia, "Electrospinning of Nanofibers: Reinventing the Wheel?" Advanced Materials, Vol. 16, No. 14, 2004, pp. 1151-1170. doi:10.1002/adma.200400719

[18] A. Greiner and J. H. Wendorff, "Electrospinning: A Fascinating Method for the Preparation of Ultrathin Fibers," Angewandte Chemie International Edition, Vol. 46, No. 30, 2007, pp. 5670-5703. doi:10.1002/anie. 200604646

[19] S. Agarwal, A. Greiner and J. H. Wendorff, "Electrospinning of Manmade and Biopolymer NanofibersProgress in Techniques, Materials, and Applications," Advanced Functional Materials, Vol. 19, No. 18, 2009, pp. 2863-2879. doi:10.1002/adfm.200900591

[20] X. Lu, C. Wang and Y. Wei, "One-Dimensional Composite Nanomaterials: Synthesis by Electrospinning and
Their Applications," Small, Vol. 5, No. 21, 2009, pp. 2349-2370. doi:10.1002/smll.200900445

[21] M. R. Abidian, D. H. Kim and D. C. Martin, "Conducting-Polymer Nanotubes for Controlled Drug Release," Advanced Materials, Vol. 18, No. 4, 2006, pp. 405-409. doi:10.1002/adma.200501726

[22] J. M. Dang and K. W. Leong, "Myogenic Induction of Aligned Mesenchymal Stem Cell Sheets by Culture on Thermally Responsive Electrospun Nanofibers," Advanced Materials, Vol. 19, No. 19, 2007, pp. 2775-2779. doi:10.1002/adma.200602159

[23] K. Yoshimatsu, L. Ye, J. Lindberg and I. S. Chronakis, "Selective Molecular Adsorption Using Electrospun Nanofiber Affinity Membranes," Biosensors and Bioelectronics, Vol. 23, No. 7, 2008, pp. 1208-1215. doi:10.1016/j.bios.2007.12.002

[24] I. S. Chronakis, A. Jakob, B. Hagstrom and L. Ye, "Encapsulation and Selective Recognition of Molecularly Imprinted Theophylline and $17 \beta$-Estradiol Nanoparticles within Electrospun Polymer Nanofibers," Langmuir, Vol. 22, No. 21, 2006, pp. 8960-8965. doi:10.1021/la0613880

[25] S. Piperno, B. T. Bui, K. Haupt and L. A. Gheber, "Immobilization of Molecularly Imprinted Polymer Nanoparticles in Electrospun Poly(Vinyl Alcohol) Nanofibers,' Langmuir, Vol. 27, No. 5, 2011, pp. 1547-1550. doi:10.1021/la1041234

[26] I. S. Chronakis, B. Milosevic, A. Frenot and L. Ye, "Generation of Molecular Recognition Sites in Electrospun Polymer Nanofibers via Molecular Imprinting," Macromolecules, Vol. 39, No. 1, 2006, pp. 357-361. doi: 10.1021/ma052091w

[27] M. S. Chen, H. F. Fan and K. C. Lin, "Kinetic and Thermodynamic Investigation of Rhodamine B Adsorption at Solid/Solvent Interfaces by Use of Evanescent-Wave Cavity Ring-Down Spectroscopy," Analytical Chemistry, Vol. 82, No. 3, 2010, pp. 868-877. doi: 10.1021/ac9020209

[28] Y. Ma, X. Jin, M. Zhou, Z. Zhang, X. Teng and H. Chen, "Chemiluminescence Behavior Based on Oxidation Reaction of Rhodamine B with Cerium(IV) in Sulfuric Acid Medium," Analytica Chimica Acta, Vol. 489, No. 2, 2003, pp. 173-181. doi:10.1016/S0003-2670(03)00756-6

[29] J. X. Yua, B. H. Li, X. M. Sun, J, Yuan and R. Chia, "Polymer Modified Biomass of Bakers Yeast For Enhancement Adsorption of Methylene Blue, Rhodamine B and Basic Magenta," Journal of Hazardous Materials, Vol. 168, No. 2-3, 2009, pp. 1147-1154. doi:10.1016/j.jhazmat.2009.02.144

[30] M. F. Hou, C. X. Ma, W. D. Zhang, X. Y. Tang, Y. N. Fan and H. F. Wan, "Removal of Rhodamine B Using Iron-Pillared Bentonite," Journal of Hazardous Materials, Vol. 186, No. 2-3, 2011, pp. 1118-1123. doi:10.1016/j.jhazmat.2010.11.110

[31] H. M. H. Gad and A. A. El-Sayed, "Activated Carbon from Agricultural By-Products for the Removal of Rhodamine-B from Aqueous Solution," Journal of Hazardous Materials, Vol. 168, No. 2-3, 2009, pp. 1070-1081. doi:10.1016/j.jhazmat.2009.02.155 
[32] H. F. Men, H. Q. Liu, Z. L Zhang, J. Huang, J. Zhang, Y. Y. Zhai and L. Li, "Synthesis, Properties and Application Research of Atrazine $\mathrm{Fe}_{3} \mathrm{O}_{4} @ \mathrm{SiO}_{2}$ Magnetic Molecularly
Imprinted Polymer," Environmental Science and Pollution Research, in Press, 2012.

doi:10.1007/s11356-011-0732-9 